\title{
Utilización de clips en la endoscopia digestiva
}

\section{¿QUÉ SON LOS CLIPS?}

Un clip es un dispositivo metálico de pequeño tamaño (8$12 \mathrm{~mm}$ ), empleado por los especialistas de digestivo durante la realización de las exploraciones endoscópicas. Este mecanismo actúa a modo de "grapa" al aplicarlo sobre los vasos sangrantes o tejidos dañados, realizando así, una compresión directa (técnica mecánica) que facilita el cierre y cicatrización de las lesiones. Dicha característica permite realizar un tratamiento sin dañar los tejidos sanos circundantes, lo que la diferencia de otras técnicas endoscópicas que emplean calor (métodos térmicos) o sustancias químicas esclerosantes (métodos de inyección).

\section{¿PARA QUÉ SE UTILIZAN?}

Las aplicaciones de los clips en la terapéutica actual son cada vez más numerosas.

Se emplean fundamentalmente para conseguir "hemostasia", evitando o deteniendo una hemorragia digestiva activa. Además de su eficacia como mecanismo hemostático, existen numerosas publicaciones que han demostrado la utilidad de los clips para cerrar perforaciones y fístulas de la pared del tracto gastrointestinal (principalmente en casos en los que la cirugía no está recomendada), servir de marcador endoscópico radiopaco (localizar lesiones mediante estudios radiológicos para posteriormente aplicar tratamientos dirigidos a la lesión) y como instrumento auxiliar para fijar accesorios (sondas de nutrición o prótesis). Recientemente incluso se han empleado en el cierre de orificios al realizar técnicas combinadas de cirugía y endoscopia como el NOTES/CETON (cirugía endoscópica a través de orificios naturales).

Sin embargo, las lesiones donde los clips han demostrado mayor efectividad son las hemorragias gastrointestinales con sangrado activo (exceptuando la hemorragia por varices esófago-gástricas), como por ejemplo las úlceras pépticas o los sangrados procedentes de arterias de pequeño tamaño. No obstante, pueden emplearse en cualquier hemorragia gastrointestinal alta o baja, independientemente de la causa. También son una herramienta rutinaria y de primera elección en la prevención del sangrado tras una polipectomía (extirpación endoscópica de un pólipo) al aplicarlos sobre la escara (cicatriz), sobre todo en pacientes que consumen medicamentos antiagregantes o anticoagulantes, donde existe un mayor riesgo de sangrado.

\section{¿CÓMO SE UTILIZAN?}

Su uso sigue el principio quirúrgico de aproximar correctamente los bordes de tejido sano de la lesión (úlceras, perforaciones, erosiones) para asegurar una adecuada reparación de los tejidos y posteriormente conseguir la cicatrización y curación.

La colocación de clips durante la endoscopia digestiva es una técnica relativamente sencilla, accesible y fácil de aprender, aunque exige un personal entrenado y familiarizado con su uso. La técnica varía en función del modelo de clip utilizado, pero todos se aplican directamente a través del canal de trabajo del endoscopio y pueden permanecer posicionados entre 2-4 semanas, desprendiéndose posteriormente sin producir ningún daño y generalmente sin que el paciente lo perciba.

Tiene un perfil de seguridad excelente y la duración del efecto terapéutico es mayor que con otras técnicas "clásicas". Su eficacia y seguridad depende de varios factores: las características de lesión, la localización del sangrado y la destreza del que los manipula. Pueden ser difíciles de posicionar adecuadamente en lesiones de gran tamaño $(>1 \mathrm{~cm})$ o con sangrado difuso, lesiones con gran cantidad de tejido cicatricial crónicas o áreas de difícil visualización durante la endoscopia (bulbo duodenal, curvadura menor gástrica o angulaciones del colon,...)

Los resultados en los estudios realizados son muy satisfactorios, con una tasa de eficacia en lesiones con sangrado activo cercana al $90 \%$, solos o en combinación con otras técnicas y una tasa de resangrado de las lesiones inferior al $10 \%$.

\section{PUNTOS PRINCIPALES}

Los clips son dispositivos metálicos, empleados en la endoscopia digestiva como herramienta terapéutica, que producen una compresión mecánica sobre las lesiones sangrantes o los tejidos lesionados. Es una técnica sencilla, eficaz y segura, que se emplea fundamentalmente para conseguir realizar un tratamiento hemostático de lesiones con sangrado activo o en la prevención (profilaxis) de la hemorragia tras realizar una polipectomía endoscópica.

Miguel Rivero Fernández y Patricia Sanz

Servicio de Gastroenterología. Hospital del Sureste. Arganda del Rey, Madrid 